\title{
Veröffentlichungen der Schweizerischen Gesellschaft für Geschichte der Medizin und der Naturwissenschaften
}

Publications de la Société suisse d'histoire de la médecine et des sciences naturelles

Noch lieferbare Bände; alle außer Band XX broschiert.

IX A. Moritzi (1806-1850), Réflexions sur l'espèce en histoire naturelle. 1842. Mit einer biographischen Einleitung nach Prof. Dr. J.Bloch und einer Würdigung Moritzis als Vorläufer Charles Darwins von Prof. Dr. Arnold Lang. 82 Seiten und 1 Tafel. 1934. Mitglieder: Fr. 25.-, Nichtmitglieder: Fr. 35.-.

$\mathrm{X}$ Wilhelm Fabry von Hilden, genannt Fabricius Hildanus, Stadtarzt in Bern von 1615 bis 1634, Von der Fürtrefflichkeit und Nutz der Anatomy. 2., erweiterte Auflage nach dem in der Stadtbibliothek von Bern befindlichen Manuskript. Herausgegeben von Prof. Dr. F. de Quervain und Dr. Hans Bloesch, unter Mitwirkung von Dr. phil. Th. de Quervain. XVI + 204 Seiten und 4 Abbildungen. 1936. Mitglieder: Fr. 25.-, Nichtmitglieder: Fr. 35.-.

XI Paul Aebischer/Eugène Olivier, L'herbier de Moudon. Un recueil de recettes médicales de la fin du XIVe siècle. Notes sur la botanique médicale au moyen-âge. 102 pages et 1 illustration. 1938. Mitglieder: Fr.25.-, Nichtmitglieder: Fr. 35.--

XV Heinrich Buess, Die historischen Grundlagen der intravenösen Injektion. Ein Beitrag zur Medizingeschichte des 17.Jahrhunderts. 208 Seiten und 4 Abbildungen. 1946. Mitglieder: Fr. 25.--, Nichtmitglieder: Fr. 35.-.

XVI Henry Nigst, Das anatomische Werk Johann Jakob Wepfers (1620-1695). 88 Seiten und 3 Abbildungen. 1946. Mitglieder: Fr. 25.-, Nichtmitglieder: Fr. 35.-.

XVII Hans Buscher, Der Basler Arzt Heinrich Pantaleon (1522-1595). XII + 76 Seiten. 1947. Mitglieder: Fr. 25.--, Nichtmitglieder: Fr. 35.--.

XVIII Gwer Reichen, Die chirurgische Abteilung des Bürgerspitals Basel zur Zeit der Antiseptik. Ein Beitrag zur Geschichte der Basler chirurgischen Klinik. 107 Seiten und 3 Porträts. 1949. Mitglieder: Fr. 25.-, Nichtmitglieder: Fr. 35.-.

XIX Sigmund Bornhauser, Zur Geschichte der Schilddrüsen- und Kropfforschung im 19.Jahrhundert (unter besonderer Berücksichtigung der Schweiz). 173 Seiten. 1951. Mitglieder: Fr. 25.-, Nichtmitglieder: Fr. 35.--

XX Heinz Balmer, Beiträge zur Geschichte der Erkenntnis des Erdmagnetismus. 892 Seiten mit 45 Abbildungen. 1956. Leinen. Mitglieder: Fr. 35.-, Nichtmitglieder: Fr. 45.-.

XXI Hans H. Walser, Zur Einführung der Äthernarkose im deutschen Sprachgebiet im Jahre 1847. 56 Seiten. 1957. Mitglieder: Fr.25.-, Nichtmitglieder: Fr.35.-.

XXII Edgar Frenk, Johann Rudolph Burkhards Syllogae Phainomenon Anatomikon, ein Einblick in das Krankengut des Zürcher Spitals vor 200 Jahren. 54 Seiten und 1 Tafel. 1958. Mitglieder: Fr. 25.-, Nichtmitglieder: Fr. 35.-.

XXIII Ambroise Boner, Suggestion et Jurisprudence. Etude du rôle de la suggestion dans les procédures légales, basée sur les résultats expérimentaux de l'Ecole de Nancy et de son prédécesseur. 36 pages et 1 planche. 1962. Mitglieder: Fr. 25.-, Nichtmitglieder: Fr. 35.--

XXIV Hans H. Walser, Hundert Jahre Klinik Rheinau, 1867-1967. Wissenschaftliche Psychiatrie und praktische Irrenpflege in der Schweiz am Beispiel einer großen Heil- und Pflegeanstalt. 78 Seiten und 12 Tafeln. 1971. Mitglieder: Fr. 25.-, Nichtmitglieder: Fr. 35.-.

XXV Henri Reverdin, Jaques-Louis Reverdin, 1842-1929. Un chirurgien à l'aube d'une ère nouvelle. 226 pages et 5 planches. 1971. Mitglieder: Fr.25.-, Nichtmitglieder: Fr. 35.-- 
Ulrich Tröhler, Der Schweizer Chirurg J.F. de Quervain (1868-1940). Wegbereiter neuer internationaler Beziehungen in der Wissenschaft der Zwischenkriegszeit. X +137 Seiten und 1 Abbildung. 1973. Mitglieder: Fr. 25.-, Nichtmitglieder: Fr. 35.--

27 Georges Schüler, Der Basler Irrenarzt Friedrich Brenner, 1809-1874. Ein Beitrag zur Geschichte der Schweizer Psychiatrie sowie zur Sozial-, Religions- und Kulturgeschichte der Stadt Basel im 19. Jahrhundert. 256 Seiten und 2 Tafeln. 1974. Mitglieder: Fr. 25.-, Nichtmitglieder: Fr. 35.--

Andreas Kleinert, Die allgemeinverständlichen Physikbücher der französischen Aufklärung. IV + 187 Seiten mit 4 Abbildungen. 1974. Mitglieder: Fr. 25.-, Nichtmitglieder: Fr. 35.--

Barbara C. Hansch-Mock, Deutschschweizerische Kalender des 19.Jahrhunderts als Vermittler schul- und volksmedizinischer Vorstellungen. VII + 415 Seiten mit 22 Abbildungen. 1976. Mitglieder: Fr.25.-, Nichtmitglieder: Fr. 35.-

30 Katharina Wäckerlin-Swiagenin, Der «Schüpfheimer Codex», ein Medizinalbuch aus dem zweiten Viertel des 15. Jahrhunderts. VIII + 187 Seiten mit 5 Abbildungen und 1 Tafel. 1976. Mitglieder: Fr.25.-, Nichtmitglieder: Fr. 35.-.

Rudolf Schmid, Die Medizin im Oberhalbstein bis zum Beginn des 20.Jahrhunderts. 108 Seiten mit 1 Abbildung. 1978. Mitglieder: Fr. 25.-, Nichtmitglieder: Fr. 35.-.

Steffen Richter, Wolfgang Pauli. Die Jahre 1918-1930. Skizzen zu einer wissenschaftlichen Biographie. 112 Seiten und 1 Abbildung. 1979. Mitglieder: Fr. 25.-, Nichtmitglieder: Fr. 35.-Huldrych M. Koelbing, Christian Sigismund Fingers Dissertation "Über den schädlichen Einflu $\beta$ von Furcht und Schreck bei der Pest» (Halle 1722). 52 Seiten mit 1 Abbildung. 1979. Mitglieder: Fr.25.-, Nichtmitglieder: Fr. 35.-.

Peter Köpp, Vademecum eines frühmittelalterlichen Arztes. 128 Seiten mit 45 Abbildungen. 1980. Mitglieder: Fr. 25.-, Nichtmitglieder: Fr. 35.-.

Adolf Faller, Wertschätzung von Stensens "Discours sur l'anatomie du cerveau» im Verlaufe von drei Jahrhunderten. 96 Seiten mit 4 Abbildungen. 1981. Mitglieder: Fr. 25.-, Nichtmitglieder: Fr. 35.-.

Carlo Prestele, Arztliche Ethik bei Fabricius Hildanus. 144 Seiten mit 1 Abbildung. 1981. Mitglieder: Fr. 25.-, Nichtmitglieder: Fr. 35.--

37 Christian Picco, Das Biochemische Institut der Universität Zürich 1931-1981. 63 Seiten mit 10 Abbildungen. 1981. Mitglieder: Fr.25.-, Nichtmitglieder: Fr. 35.-.

38 Hans Konrad Iselin, Zur Entstehung von C.G.Jungs «Psychologischen Typen». Der Briefwechsel zwischen C. G. Jung und Hans Schmid-Guisan im Lichte ihrer Freundschaft. 152 Seiten mit 6 Abbildungen. 1982. Mitglieder: Fr. 25.-, Nichtmitglieder: Fr. 35.--

Roger Neiger, Jakob Wyrsch (1892-1980). Leben und Werk. VI + 114 Seiten. 1985. Mitglieder: Fr. 25.-, Nichtmitglieder: Fr. 35.--

Andreas-Holger Maehle, Johann Jakob Wepfer (1620-1695) als Toxikologe. Die Fallstudien und Tierexperimente aus seiner Abhandlung über den Wasserschierling (1679). 224 Seiten. 1987. Mitglieder: Fr.35.-, Nichtmitglieder: Fr. 42.-.

Preisstand 1988. Änderungen vorbehalten

Verlag Sauerländer, Postfach, CH - 5001 Aarau 